\title{
Timing of the accreting millisecond pulsar IGR J17511-3057
}

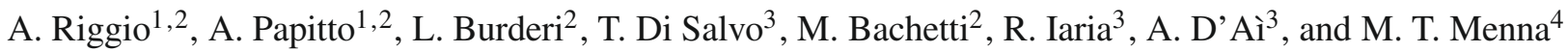 \\ 1 INAF/Osservatorio Astronomico di Cagliari, località Poggio dei Pini, strada 54, 09012 Capoterra, Italy \\ e-mail: ariggio@oa-cagliari.inaf.it \\ 2 Università di Cagliari, Dipartimento di Fisica, SP Monserrato-Sestu km 0,7, 09042 Monserrato (CA), Italy \\ 3 Dipartimento di Scienze Fisiche e Astronomiche, Università di Palermo, via Archirafi 36, 90123 Palermo, Italy \\ 4 Osservatorio Astronomico di Roma, Sede di Monteporzio Catone, via Frascati 33, 00040 Roma, Italy
}

Received 25 February 2010 / Accepted 23 October 2010

\section{ABSTRACT}

\begin{abstract}
Context. Timing analysis of accretion-powered millisecond pulsars (AMPs) is a powerful tool for probing the physics of compact objects. The recently discovered IGR J17511-3057 was the twelfth discovered of the 13 AMPs known. The Rossi XTE satellite provided an extensive coverage of the 25 days-long observation of the source outburst.

Aims. Our goal is to investigate the complex interaction between the neutron star magnetic field and the accretion disk by determining the angular momentum exchange between them. The presence of a millisecond coherent flux modulation allows us to investigate this interaction from the study of pulse arrival times. To separate the neutron star proper spin frequency variations from other effects, a precise set of orbital ephemeris is mandatory.

Methods. Using timing techniques, we analysed the pulse phase delays by fitting differential corrections to the orbital parameters. To remove the effects of pulse phase fluctuations, we applied the timing technique that had been already successfully applied to the case of an another AMP, XTE J1807-294.

Results. We report a precise set of orbital ephemeris. We demonstrate that the companion star is a main-sequence star. We find pulse phase delay fluctuations on the first harmonic with a characteristic amplitude of about 0.05 , similar to those also observed for the AMP XTE J1814-338. For the second time, an AMP shows a third harmonic detected during the entire outburst. The first harmonic phase delays exhibit a puzzling behaviour, while the second harmonic phase delays clearly spin-up. The third harmonic also shows a spin-up, although not highly significant $(3 \sigma$ c.l.). The presence of a fourth harmonic is also reported. If we assume that the second harmonic is a good tracer of the spin frequency of the neutron star, we infer a mean spin frequency derivative for this source of $1.65(18) \times 10^{-13} \mathrm{~Hz} \mathrm{~s}^{-1}$.

Conclusions. To interpret the pulse phase delays of the four harmonics, we apply the disk threading model, but obtain different and incompatible $\dot{M}$ estimates for each harmonic. In particular, the phase delays of the first harmonic are heavily affected by phase noise, and consequently, on the basis of these data, it is not possible to derive a reliable estimate of $\dot{M}$. The second harmonic gives a $\dot{M}$ consistent with the flux assuming that the source is at a distance of $6.3 \mathrm{kpc}$. The third harmonic gives a lower $\dot{M}$ value, with respect to the first and second harmonic, and this would reduce the distance estimate to $3.6 \mathrm{kpc}$.
\end{abstract}

Key words. stars: neutron - stars: magnetic field - pulsars: general - pulsars: individual: IGR J17511-3057 - X-rays: binaries

\section{Introduction}

Accretion-powered millisecond pulsars (hereafter AMPs) are transient low mass X-ray binaries, that exhibit a coherent modulation of their X-ray fluxes with periods of the order of few milliseconds. In the recycling scenario, AMPs are seen as the progenitors of millisecond radio pulsars (see e.g. van den Heuvel 1984), the accretion process being responsible for the spinning up of the neutron star (hereafter NS) to millisecond periods.

The AMP IGR J17511-3057 was discovered by INTEGRAL on 12 September, 2009 during a Galactic bulge monitoring programme (Baldovin 2009). Although very close to the previously known AMP XTE J1751-305, the source position measured by INTEGRAL implied that it was a newly discovered X-ray source. The observation of a coherent modulation of the X-ray flux in the data from a ToO observation performed by the Rossi X-ray Timing Explorer (hereafter $R X T E$ ) with a period of about $4 \mathrm{~ms}$ permitted Markwardt et al. (2009b) to classify IGR J17511-3057 as an AMP and confirmed it as a new transient X-ray source. Altamirano et al. (2010) reported the presence of burst oscillations at the NS frequency. An analysis of a Chandra observation by Nowak et al. (2009) gave the best source position with an uncertainty of 0.6". IGR J17511-3057 was observed by Swift and the data analysed by Bozzo et al. (2010), which provided a description of the X-ray spectrum. Torres et al. (2009) reported a possible near-infrared counterpart. Considering a ToO XMMNewton observation, Papitto et al. (2010) obtained a set of orbital parameters and performed a detailed analysis of the X-ray spectrum. Riggio et al. (2009), analysing a RXTE observation, refined these orbital parameters. Miller-Jones et al. (2009) set an upper limit on the radio emission. Surprisingly, another transient X-ray source (XTE J1751-305) went into outburst very near the position of IGR J17511-3057 on 7 October, 2009 (Chenevez et al. 2009); its pulsations were detected by RXTE (Markwardt et al. 2009a) while observing IGR J17511-3057, which faded beneath its detection threshold on 8 October, 2009.

In this paper, we present a detailed timing analysis of the RXTE ToO observation of the source IGR J17511-3057.

\section{Observation and data analysis}

We analyse an RXTE observation of IGR J17511-3057. In particular, we use data from the PCA (proportional counter array) 


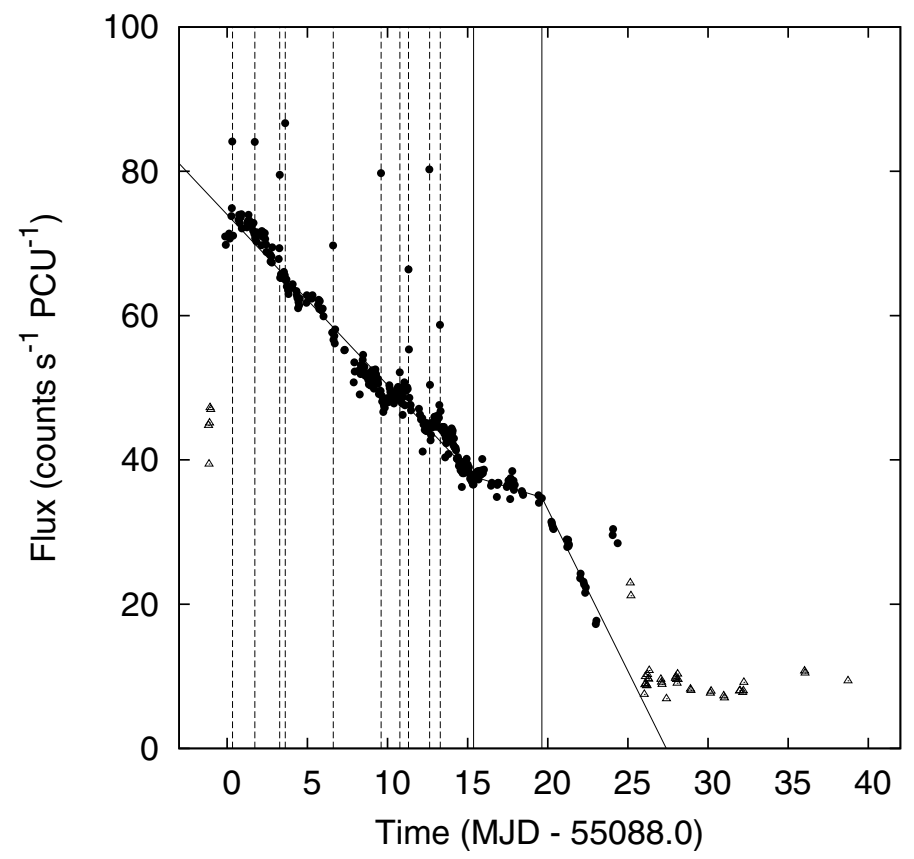

Fig. 1. PCU 2 count rate $(2-25 \mathrm{keV})$, subtracted of its background, is reported as a function of time during the outburst. The superimposed model represents the best fit using a piecewise linear function. The abrupt flux increase at the end of the outburst is due to the onset of an outburst from the AMP XTE J1751-305. The dashed vertical lines correspond to the type-I bursts present in the observation, while the continuous vertical lines correspond to the slope change, which depends on the model used to describe the count rate. The filled circles are relative to the ObsId 94041, while the triangles are the XTE J1751-305 observation (ObsId 94042).

instrument board on the RXTE satellite (ObsId P94041 and P94042). We used data collected in event packing mode, with time and energy resolutions of $122 \mu$ s and 64 energy channels, respectively. We selected data in the energy range $2-25 \mathrm{keV}$ to maximise the signal-to-noise ratio, since above $\sim 20 \mathrm{keV}$ the background is the dominant signal. The X-ray flux follows a piecewise linear decay as showed in Fig. 1, with a peak flux of 70 counts $\mathrm{s}^{-1} \mathrm{PCU}^{-1}$. The re-brightening visible in Fig. 1, 24 days after the start of the observation, is due to the AMP XTE J1751-305 in the field of view of RXTE going into outburst. The analysed data cover the time span from 12 September 2009 (MJD 55 086.8) to 22 October 2009 (MJD 55 126.8). We corrected the photon arrival times for the motion of the Earthspacecraft system with respect to the Solar System barycentre and reported them as barycentric dynamical times at the Solar System barycentre using the faxbary tool (DE-405 solar system ephemeris). We used the Chandra source position reported by Nowak et al. (2009), which is reported in Table 1. The uncertainty in the source position quoted by Nowak et al. (2009) is $0.6^{\prime \prime}$, at the $1 \sigma$ confidence level.

\subsection{Derivation of the orbital ephemeris}

To obtain a first estimate of the mean spin frequency, we produced a power spectrum from the first data file of the ObsID 94041-01-01-00. This was obtained averaging 53 power spectra 64-seconds long each and using a bin size of $2^{-11} \mathrm{~s}$. We found a strong signal at $\sim 244.81 \mathrm{~Hz}$, in good agreement with the value reported by Markwardt et al. (2009b). We divided the observation into time intervals of about $400 \mathrm{~s}$ each and performed
Table 1. Orbital and spin parameters for IGR J17511-3057.

\begin{tabular}{lc}
\hline \hline Parameter & Value \\
\hline RA (J2000) (Nowak et al. 2009) & $17^{\mathrm{h}} 51^{\mathrm{m}} 08^{\mathrm{s}} .66$ \\
Dec (J2000) (Nowak et al. 2009) & $-30^{\circ} 57^{\prime} 41^{\prime \prime} 0$ \\
Orbital period $P_{\text {orb }}(\mathrm{s})$ & $12487.5121(4)$ \\
Projected semi-major axis, $a_{x} \sin i(\mathrm{lt}-\mathrm{ms})$ & $275.1952(18)$ \\
Ascending node passage, $T^{\star}(\mathrm{MJD})$ & $55088.0320279(4)$ \\
Eccentricity, $e$ & $<3 \times 10^{-5}$ \\
Mass function ${ }^{(1)}, f_{x}\left(M_{\odot}\right)$ & $1.070854(21) \times 10^{-3}$ \\
Reference epoch, $T_{0}(\mathrm{MJD})$ & 55088.0 \\
Mean spin frequency, $v_{0}(\mathrm{~Hz})$ & $244.83395156(7)$ \\
\hline Constant $\dot{v}$ model best-fit parameters & $1.74(238.5 / 137)$ \\
$\chi_{r}^{2}\left(\chi^{2} /\right.$ d.o.f.) & $244.83395145(9)$ \\
Spin frequency at $T_{0}, v_{0}(\mathrm{~Hz})$ & $1.45(16) \times 10^{-13}$ \\
Spin frequency derivative, $\dot{v}\left(\mathrm{~Hz} \mathrm{~s} \mathrm{~s}^{-1}\right)$ & \\
\hline Physical model best - fit parameters & $1.70(232.8 / 137)$ \\
$\chi_{r}^{2}\left(\chi^{2} /\right.$ d.o.f.) & $244.83395145(9)$ \\
Spin frequency, $v_{0}(\mathrm{~Hz})$ & $0.92(10) \times 10^{-9}$ \\
Accretion rate at $T_{0}\left(M_{\odot}\right.$ year $\left.^{-1}\right)$ & \\
\hline
\end{tabular}

Notes. Errors are intended to be at $1 \sigma$ c.l., upper limits are given at 95\% confidence level. Times are given with respect to the barycentre of the Solar System (TDB). Best-fit spin parameters are derived for both hypotheses of a constant spin-up and flux dependent spin-up, and the uncertainties in the given values of $v, \dot{v}$, and $\dot{M}_{\max }$ include systematics due to the uncertainties in the source position (see text). Here we report only the second harmonic best-fit spin parameters. ${ }^{(1)}$ This value was obtained using the latest available measure of $G, c$ (http://physics. nist.gov/cuu/Constants/) and $M_{\odot}$ (http://nssdc.gsfc.nasa. gov/planetary/factsheet/sunfact.html).

an epoch folding search on each data interval around the averaged spin period with a period resolution of $4 \times 10^{-9} \mathrm{~s}$. For each time interval, we obtained an estimate of the best spin period. We excluded all the intervals for which the maximum in the $\chi^{2}$ curve was not significant ( $3 \sigma$ c.l.), according to the criterion stated by Leahy et al. (1983). A sinusoidal Doppler modulation of the spin period due to the source motion in the binary system was evident. We fitted the Doppler frequency shifts with the formula

$v(t)=v_{0}+\dot{v}\left(t-T_{0}\right)-\frac{2 \pi v_{0} A}{P_{\text {orb }}} \cos l(t)$,

where $v_{0}$ is the spin frequency at the time $T_{0}, \dot{v}$ is the spin frequency derivative, $A$ is the ratio of the projected orbit semi-major axis to the speed of light, and $l(t)=2 \pi\left(t-T_{\star}\right) / P_{\text {orb }}$, where $T_{\star}$ is the time of passage through the ascending node and $P_{\text {orb }}$ is the orbital period. With a reduced $\chi^{2}$ (hereafter $\chi_{r}^{2}$ and defined as $\chi^{2} /$ d.o.f.) of $0.59(532.2 / 898)$, we obtained a first set of orbital parameters and a much more reliable estimate of the barycentric spin frequency.

Using this preliminary orbital solution, we analysed the pulse phase delays to get a more precise estimate of the orbital and spin parameters. We epoch-folded data across time intervals of about $1500 \mathrm{~s}$ using 32 phase channels. An example of the folded pulse profile is shown in Fig. 2. A harmonic decomposition of each pulse profile up to the fourth harmonic was necessary. To do that we fitted each normalised pulse profile using the expression

$f(\phi)=1+\sum_{n=1}^{4} a_{n} \sin \left(2 n \pi\left(\phi-\phi_{n}\right)\right)$,

where $a_{1}, a_{2}, a_{3}$, and $a_{4}$ are the sinusoidal semi-amplitudes (hereafter fractional amplitudes) of the first, second, third, and 


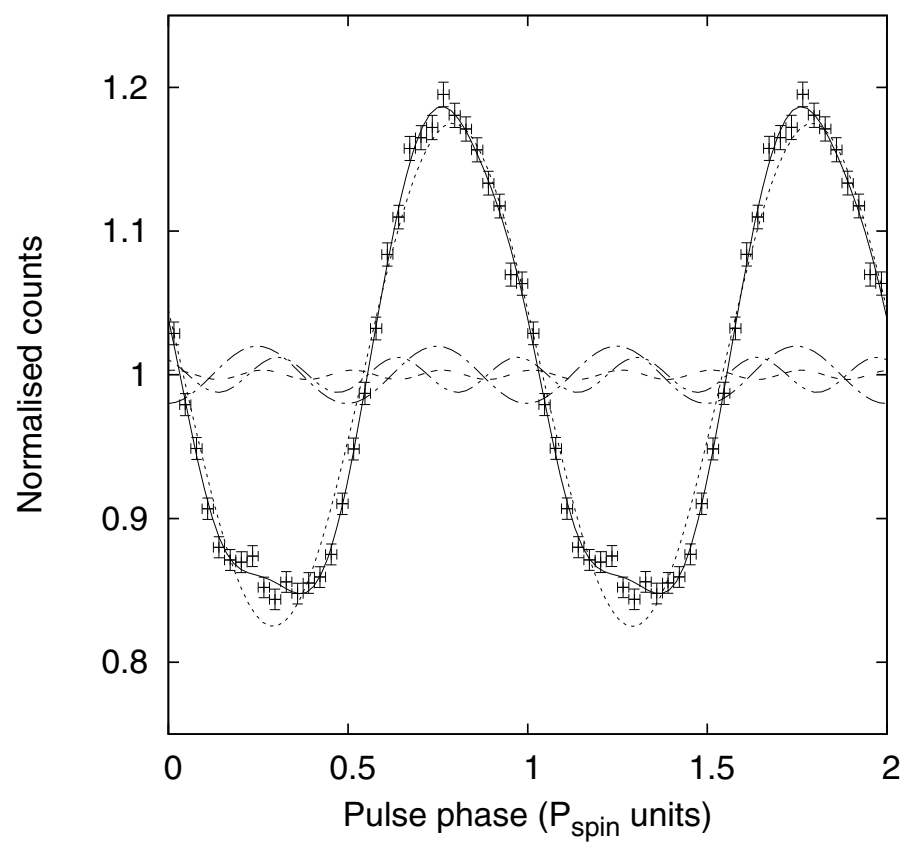

Fig. 2. A folded pulse profile is shown. For clarity, two spin cycles are plotted. The continuous line is the best-fit using first, second, and third harmonics. We also report the single contribution to the profile of the first harmonic (dotted line), second harmonic (dot-dashed line), third harmonic (bi-dot dashed line), and fourth harmonic (dashed line).

fourth harmonics, respectively, and $\phi_{1}, \phi_{2}, \phi_{3}$, and $\phi_{4}$ their corresponding phases. We rejected the pulse phase delays for which the following two conditions were not both satisfied: i) the signal is not detected at least at a $3 \sigma$ confidence level (Leahy et al. $1983)$; ii) the best-fit fractional amplitude had to be at least $3 \sigma$ from the zero level $\left(a_{i} / \delta a_{i} \geq 3\right)$.

We first tried to fit the pulse phase delays with a polynomial to describe the pulse phase delay long-term fluctuations plus the usual formula $\phi_{\text {orb }}(t)$, describing the pulse phase residuals due to differential corrections to the initial orbital parameter estimates (Deeter et al. 1981; see e.g. Riggio et al. 2007)

We attempted to describe the phase fluctuations using a polynomial up to the ninth degree to fit obtaining a $\chi_{r}^{2}=$ 6.11(1834.6/300), which is formally unacceptable.

Owing to the presence of these phase fluctuations, we decided to apply the timing technique described in Riggio et al. (2007) to separate the orbital modulation from the phase fluctuations, to obtain more accurate estimates of the orbital parameters.

Following Riggio et al. (2007), we define the pulse phase differences as

$\Delta \phi_{\text {orb }}\left(t_{i}\right)=\phi_{\text {orb }}\left(t_{i+1}\right)-\phi_{\text {orb }}\left(t_{i}\right)$.

We excluded all the points for which $t_{i+1}-t_{i}>P_{\text {orb }}$ to optimise the filter efficiency (Riggio et al. 2007).

We iterated the process until convergence. In the last iteration (see Fig. 3), $\chi_{r}^{2}=1.43(398 / 279)$, which represents a far closer fit than the previous approach. The orbital ephemeris bestfit results are reported in Table 1, where the errors have been multiplied by the factor $\sqrt{\chi_{r}^{2}}$ (Bevington \& Robinson 2003).

The values obtained are in perfect agreement with those reported by Papitto et al. (2009) from an analysis of a XMMNewton observation and by Riggio et al. (2009) on the basis of RXTE data covering a shorter time interval. The measured uncertainties in the orbital parameters are a factor of two smaller

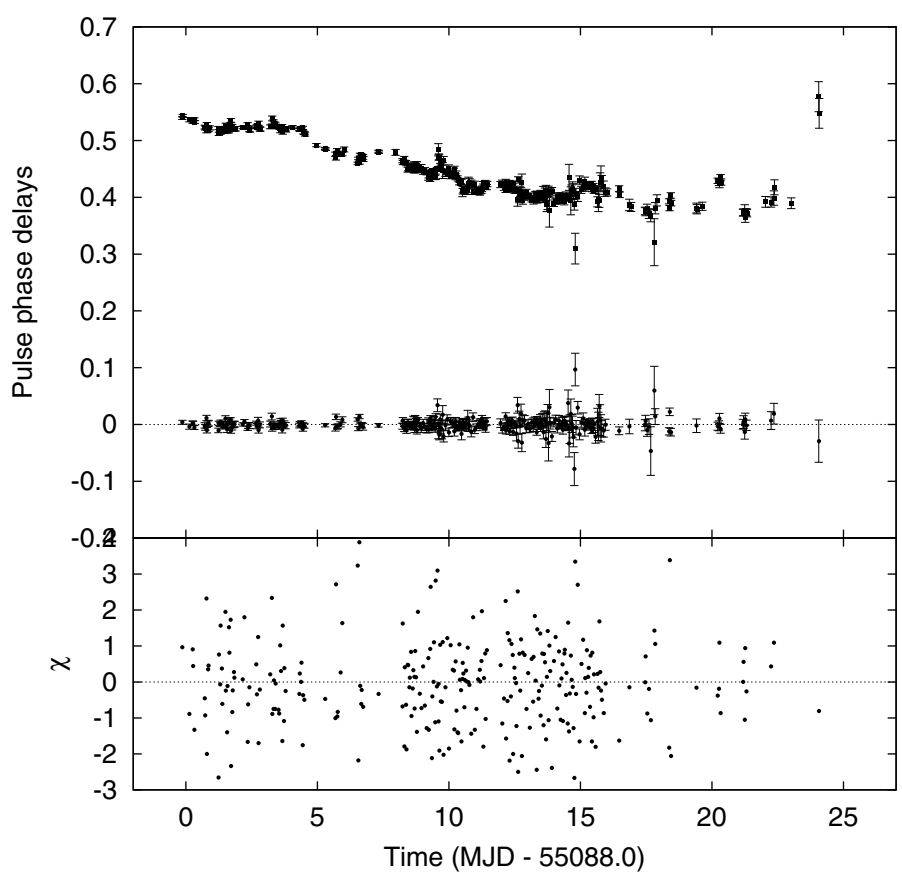

Fig. 3. In the top panel of this figure the pulse phase delays (filled squares) and the corresponding pulse phase delay differences (filled circles) of the data corrected with the orbital ephemeris obtained from the fit to the frequency Doppler shifts are reported. Each pulse phase point is obtained by fitting the folded pulse profile over about $1500 \mathrm{~s}$ of data. It is evident that there is no trace of this fluctuation in the pulse phase delay differences, giving a striking confirmation of the goodness of the method. In the bottom panel, we report the residuals (in $\sigma$ units) with respect to the best fit orbital solution derived from the pulse phase delay differences using the timing technique described in the text.

than the best-fit orbital solution previously given by Riggio et al. (2009). We also note that the orbital period we derive here has a relative uncertainty as small as $0.03 \%$.

To fit the pulse phase delays with a physically meaningful torque that takes into account the decreasing X-ray flux (hence $\dot{M}$, where the X-ray flux is a good tracer of the mass accretion rate onto the NS), we need to describe the flux evolution during the outburst, i.e. both the bolometric flux at the peak of the outburst and the shape of the light curve.

To estimate the bolometric flux of the source at the peak of the outburst, we considered the spectrum collected by the PCA in Standard 2 Mode (129 energy channels recorded at a time resolution of $16 \mathrm{~s}$ ) during Obs. 94041-01-01-04, which started on 55 089.283 MJD and had a total exposure of $10480 \mathrm{~s}$. We considered only data taken by the PCU2 to avoid crosscalibration problem between PCUs. We selected only events detected in its top Xenon layer to maximise the signal-to-noise ratio (Jahoda et al. 2006). The background was modelled using the bright source model, which is appropriate for sources emitting $>40 \mathrm{c} \mathrm{s}^{-1} \mathrm{PCU}^{-1}$. We used the latest version (11.7) of the PCA response matrix generator, restricting ourselves to the $3-50 \mathrm{keV}$ band and adding a systematic error of $0.5 \%$ to spectral counts ${ }^{1}$. As observed for other AMPs, the X-ray emission of IGR J17511-3057 is dominated by power-law-like emission extending to high $(>50 \mathrm{keV})$ energies. We modelled this using a simple thermal Comptonisation model (nthcomp,

1 N. Shaposhnikov, K. Jahoda, C. B. Markwardt 2009, http:// www . universe .nasa.gov/xrays/programs/rxte/pca/doc/rmf/ pcarmf-11.7/ 
Zdziarski et al. 1996; Życki et al. 1999), fixing the temperature of the hot electrons to $100 \mathrm{keV}$. Given the poor coverage of the PCA at low energies, we also constrained the absorption column to be $n H=1 \times 10^{22} \mathrm{~cm}^{-2}$, as suggested by an XMM-Newton observation performed during the same outburst (Papitto et al. 2010). A $6.6 \mathrm{keV}$ emission line was also added to model residuals in the iron range, though this feature is probably due to the contamination of the Galactic ridge in the field of view of the PCA (Markwardt et al. 2009b). The reduced chisquare of the fit is good $\left(\chi_{r}^{2}=64.3 / 72\right)$. The unabsorbed flux we detect in the 3-50 keV band is $1.14(1) \times 10^{-9} \mathrm{erg} \mathrm{cm}^{-2} \mathrm{~s}^{-1}$, which extrapolated to the $0.5-200 \mathrm{keV}$ band infers a flux there of $\sim 2 \times 10^{-9} \mathrm{erg} \mathrm{cm}^{-2} \mathrm{~s}^{-1}$. Under the hypothesis that emission is isotropic, this corresponds to a bolometric luminosity of $L_{\mathrm{x}} \sim 1.5 \times 10^{37} d_{8}^{2} \mathrm{erg} \mathrm{s}^{-1}$, where $d_{8}$ is the distance to the source in units of $8 \mathrm{kpc}$. We note that an upper limit of $10.6 \mathrm{kpc}$ on the source distance was set by Bozzo et al. (2010), by insisting that the burst peak luminosity does not exceed the Eddington limit, while Altamirano et al. (2010), from the analysis of the type-I bursts observed by RXTE and Swift found an upper limit of $6.9 \mathrm{kpc}$. On the basis of the spectral analysis of XMM-Newton data, Papitto et al. (2010) also gave a lower limit of $\simeq 7 \mathrm{kpc}$, which was, however, based on some assumptions. Moreover, as the source is only a few degrees away from the Galactic centre and its X-ray emission is not heavily absorbed ( $\mathrm{nH} \sim 10^{22} \mathrm{~cm}^{-2}$ ), it is highly probable that the distance does not exceed $8 \mathrm{kpc}$. Assuming that $L_{\mathrm{X}}=\epsilon G M \dot{M} / R$ with $\epsilon \simeq 1$, we eventually deduce a peak mass accretion rate of the order of $\simeq 1.5 \times 10^{-9} d_{8}^{2} M_{\odot} \mathrm{yr}^{-1}$, which is the estimate we use in the following to compare the dynamical estimates of $\dot{M}$ from the timing analysis.

To describe the light curve shape, we chose to fit it with a piecewise linear function composed of three segments, as shown in Fig. 1. We fitted only the first 23 days of data since the subsequent data are affected by the concomitant XTE J1751-305 outburst. We modelled each of the three intervals with a function $c_{i}(t)=c_{i}\left(1-\left(t-T_{i}\right) / \tau_{i}\right), T_{i} \leq t<T_{i+1}$, where $c_{i}$ is the count rate at $t=T_{i}$ and $\tau_{i}$ the linear decay timescale for the $i$ th piece. For the sake of simplicity, we wrote this piecewise function as $c_{0} f(t)$, where $c_{0}$ is the count rate at the peak. The best-fit result is reported in Fig. 1.

\subsection{Timing analysis}

The spin frequency evolution in AMPs is thought to be driven by the accretion process. Matter falling from the accretion disk onto the NS transfers its angular momentum to the NS, which is spunup to millisecond spin periods. However, as was evident from the first attempts (Ghosh et al. 1977), the magnetic field-accretion disk interaction can exert a negative torque onto the NS, spinning it down. This is called the threaded disk model. Owing to the complexity of the problem, the details of the NS magnetospheredisk interaction are still not well understood. Three examples of AMPs that spin-down while accreting have been reported (Galloway et al. 2002; Burderi et al. 2006; Papitto et al. 2008).

As already observed in another two AMPs (see e.g. Burderi et al. 2007, for SAX J1808.4-3658; and Riggio et al. 2008, for XTE J1807-294), for this source the first harmonic is also dominated by fluctuations and then unusable for our scope. We note that the two AMPs cited above (SAX J1808.4-3658 and XTE J1807-294) display a second harmonic with a more regular behaviour.
An alternative interpretation of the pulse frequency derivatives was given by Hartman et al. (2008), who suggested that the red timing noise affecting the pulse phase delays can mimic a spin frequency derivative. Patruno et al. (2009) attempted to demonstrate that the pulse phase delays are correlated with the $\mathrm{X}$-ray flux, rather than the genuine spin evolution of the source, produced by the motion of the hot-spot related to the flux. Unfortunately, this correlation is not clear, even in the sign, in all AMPs and differs, in the same sources (see e.g. SAX J1808.43658 and XTE J1807-294), for each harmonic component, as noted by Patruno et al. (2009). We tested this hypothesis using the method described in Patruno et al. (2009), adopting a constant spin frequency model to derive the pulse phase residuals. For the best-fit solution that maximises the linear correlation between the phase residuals and flux, we obtained $\chi_{r}^{2} \simeq 23$ (4625/201 d.o.f.), indicating that, for this source, the pulse phase residuals of the first harmonic cannot be ascribed to flux variation and/or fluctuations.

In the following, we will work under widely accepted hypothesis that the pulse frequency is the NS spin frequency. We analyse the pulse phase delays and apply to them a disk threading model to derive the $\dot{M}$ and compare this value with that obtained from the spectral analysis of the same data.

We epoch-folded data across time intervals of about $3.0 \mathrm{ks}$ (one pulse profile per data file) and 32 phase bins. A third harmonic was detected throughout the outburst. A fourth harmonic was also detected in the first 15 days of the outburst. In Figs. 4-7 the first, second, third, and fourth harmonic pulse phase delays are reported, respectively. The fractional amplitudes, as defined in Eq. (2), were corrected to take into account the instrumental background $\left(\sim 11\right.$ counts $\mathrm{s}^{-1} \mathrm{PCU}^{-1}$ in the $2-25 \mathrm{keV}$ energy band) and the background due to the presence of the Galactic ridge in the field of view of RXTE (Markwardt et al. 2009b). To estimate this supplementary background, we used the observations of the AMP XTE J1751-305 when both sources went to quiescence, in particular the observations from MJD 55115.400 to 55126.745 , which represent a total exposure time of $33.7 \mathrm{ks}$. Owing to the low count rate, we used the faint background model, obtaining a count-rate of $\sim 7$ counts $\mathrm{s}^{-1} \mathrm{PCU}^{-1}$ in the same energy band. In Fig. 8, the fractional amplitudes for the four harmonics are reported.

The mean spin frequency reported in Table 1 is obtained by fitting the first harmonic pulse phase delays with a constant spin frequency model. The value obtained in this way for the spin frequency is $v=244.8339515569(24) \mathrm{Hz}$. However, the systematic effects due to the uncertainty of $0.6^{\prime \prime}$ (Nowak et al. 2009) in the source position brings the mean spin frequency error to $7 \times 10^{-8} \mathrm{~Hz}$ (see Burderi et al. 2007).

\subsubsection{First harmonic}

We started fitting the first harmonic pulse phase delays with a constant spin frequency derivative to estimate the mean spin frequency derivative. From the fit we obtained $\dot{v}=$ $-7.0(9) \times 10^{-14} \mathrm{~Hz} \mathrm{~s}^{-1}$ with a $\chi_{r}^{2}=18.51(3869 / 209)$, clearly unacceptable.

To improve this result, we used the threaded disk model previously applied to the AMPs by Rappaport et al. (2004). According to this model, the net torque acting on an AMP is

$\tau(t)=2 \pi I \dot{v}(t)=\dot{M}(t) \sqrt{G M R_{\mathrm{c}}}-\frac{\mu^{2}}{9 R_{\mathrm{c}}^{3}}$, 


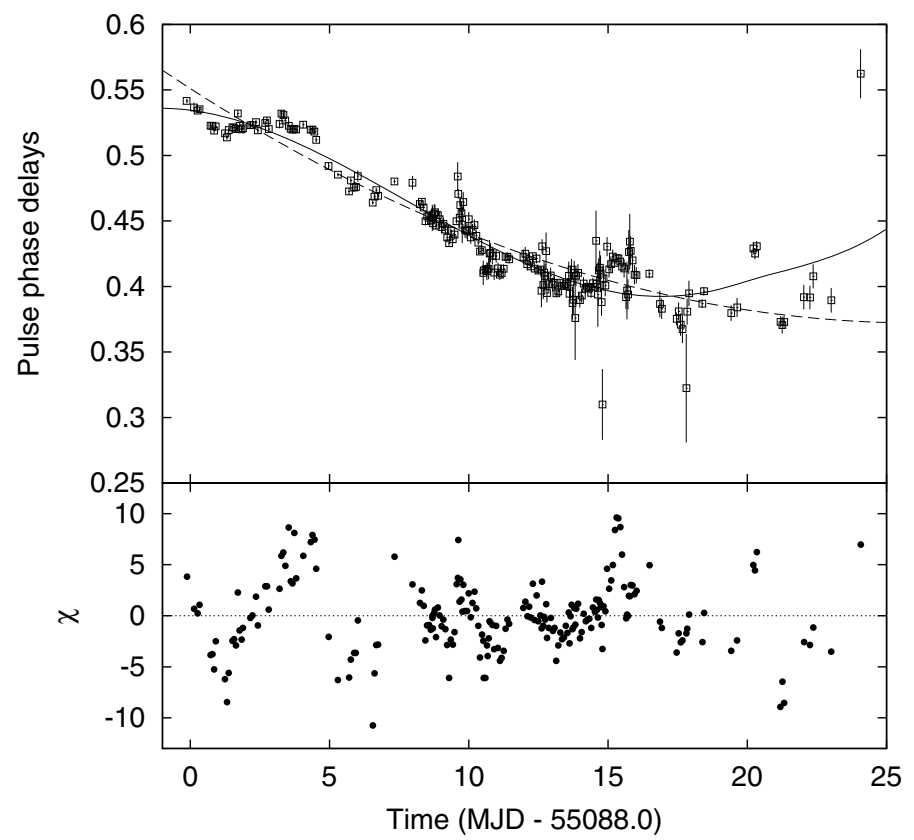

Fig. 4. In the top panel, we show the first harmonic pulse phase delays and the best-fit curves, considering a constant spin frequency derivative (dashed line) and the disk threading model proposed by Rappaport et al. (2004). In the bottom panel, we show the residuals (in $\sigma$ units) of the first harmonic with respect to the best-fit model given by the threaded disk.

where $I$ is the NS moment of inertia, $\dot{M}$ is the mass accretion rate, $M$ is the mass of the NS, $\mu$ is the magnetic dipole moment of the NS, and $R_{\mathrm{c}}$ the co-rotation radius.

To apply this model to our data, we required an expression for $\dot{M}(t)$. We then assumed that the bolometric luminosity $L(t)$ is a good tracer of $\dot{M}$. For the hypothesis that the spectral variation during the outburst is insignificant, it is possible to assume that $L(t)$ is proportional to the background subtracted count rate. We can then write $\dot{M}(t)=\dot{M}_{\max } f(t)$, where $\dot{M}_{\max }$ is the maximum accretion rate corresponding to the flux peak and $f(t)$ is the functional form of the count rate previously derived.

An expression for $\dot{v}$ as a function of $\dot{M}$ can easily be derived from Eq. (4) to be

$\dot{v}(t)=\left[1.427 \frac{m^{2 / 3} P_{-3}^{1 / 3} \dot{M}_{-10}}{I_{45}} f(t)-5.232 \frac{\mu_{26}^{2}}{m I_{45} P_{-3}^{2}}\right] \times 10^{-14} \mathrm{~Hz} \mathrm{~s}^{-1}$,

where $m$ is the NS mass in units of $M_{\odot}, \dot{M}_{-10}$ is the maximum mass accretion rate in units of $10^{-10} M_{\odot} \mathrm{y}^{-1}, P_{-3}$ the spin period in units of $10^{-3} \mathrm{~s}$, and $I_{45}$ the NS moment of inertia in $10^{45} \mathrm{~g} \mathrm{~cm}^{2}$. In this work, we adopted the FPS (Friedman Pandharipande Skyrme, see Friedman \& Pandharipande 1981; Pandharipande \& Ravenhall 1989) equation of state for which, fixing NS mass to be $M=1.4 M_{\odot}$, we obtain a radius of $R_{\mathrm{NS}}=1.14 \times 10^{6} \mathrm{~cm}$ and a moment of inertia $I=1.29 \times 10^{45} \mathrm{~g} \mathrm{~cm}^{2}$.

The pulse phase delay formula used for the fit is obtained by doubly integrating Eq. (5) with respect to time (see e.g. Burderi et al. 2007).

From the fit of the first harmonic, we obtained a magnetic dipole strength of $\mu=1.64(7) \times 10^{27} \mathrm{G} \mathrm{cm}^{3}$ and $\dot{M}_{\max }=$ $5.7(6) \times 10^{-9} M_{\odot} \mathrm{y}^{-1}$, with a $\chi_{r}^{2}=12.36(2570 / 208)$, which is still unacceptable. The results are reported in Fig. 4. This large $\chi_{r}^{2}$ is clearly due to fluctuations in the pulse phase delays, as it

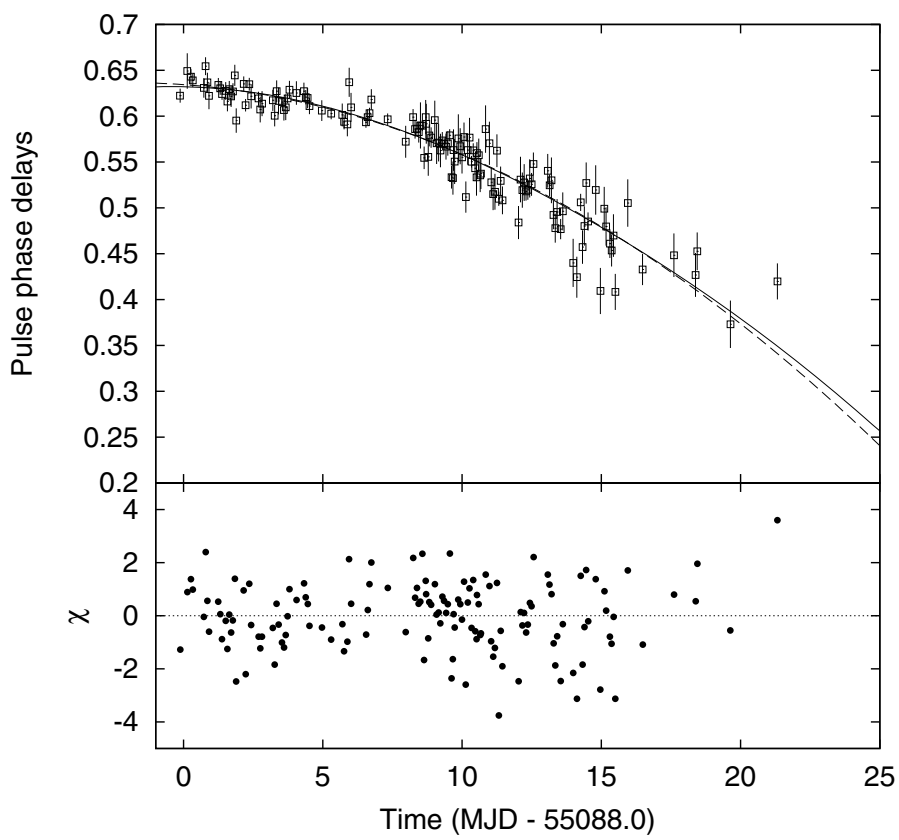

Fig. 5. In the top panel of this figure, the second harmonic pulse phase delays are shown. The dashed and the continuous lines are the second harmonic best-fit curves considering a constant spin frequency derivative and the physical model considering the material torque proportional to the flux, respectively. In the bottom panel, the residuals (in $\sigma$ units) of the second harmonic with respect the constant spin frequency derivative are shown.

is possible to see in the best-fit residuals reported in Fig. 4 (bottom panel). As anticipated, we unsuccessfully tried to interpret it with the model suggested by Patruno et al. (2009). The origin of these fluctuations remains unexplained.

\subsubsection{Second harmonic}

For the second harmonic that is less affected by phase noise (Burderi et al. 2006; Riggio et al. 2008), we repeated the fitting procedure using these phase delays. In the constant spin frequency derivative case, we obtained $\dot{v}=1.45(16) \times 10^{-13} \mathrm{~Hz} \mathrm{~s}^{-1}$ with a $\chi_{r}^{2}=1.74(238.5 / 137)$. The best-fit curve is reported in Fig. 5. We adopted the same disk threading expression used for the first harmonic to describe the second harmonic. In this case, we had to assume that $\mu=0$ since $\mu$ and $\dot{M}_{\max }$ strongly correlate. In this case, therefore, the derived value of $\dot{M}_{\max }$ has to be considered as a lower limit to the mass accretion rate at the peak of the outburst, since the value of the magnetic moment higher than zero will give a higher value for $\dot{M}_{\max }$. The best-fit results are reported in Table 1 . In this case, the best-fit value of $\dot{M}_{\max }=$ $0.92(10) \times 10^{-9} M_{\odot}$ year $^{-1}$, with $\chi_{r}^{2}=1.70(232.8 / 137)$, is in good agreement with our estimate of the bolometric flux for a source distance of about 6.3(3) kpc.

\subsubsection{Third harmonic}

Since in this source, the third harmonic is significantly detected in nearly the whole outburst we proceeded with the same method used for the first and second harmonic. From the fit with the constant spin frequency derivative, we obtained $v=244.83395151(6) \mathrm{Hz}$ and $\dot{v}=4.8(1.4) \times 10^{-14} \mathrm{~Hz} \mathrm{~s}^{-1}$ with a $\chi_{r}^{2}=1.58(159.8 / 101)$. The best-fit curve is reported in Fig. 6. We applied the threaded disk model to describe the third 


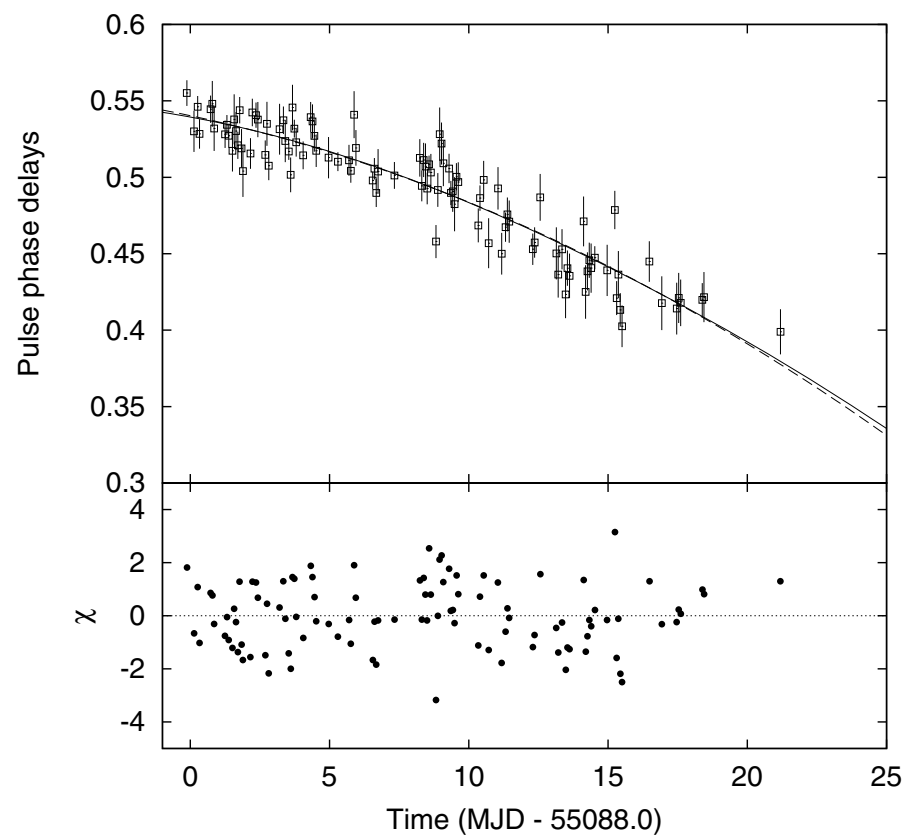

Fig. 6. In the top panel of this figure, we report the third harmonic pulse phase delays and its best-fit curves considering a constant spin frequency derivative (dashed line) and the physical model considering the material torque proportional to the flux (continuous line). In the bottom panel, we show the residuals in units of $\sigma$ with respect to the constant spin frequency derivative model.

harmonic and, as already done for the second harmonic, we fixed $\mu=0$ since $\mu$ and $\dot{M}_{\max }$ correlate in the fit. The obtained bestfit values are $\dot{M}_{\max }=3.1(9) \times 10^{-10} M_{\odot}$ year $^{-1}$ for the peak mass accretion rate and $v=244.83395150(6) \mathrm{Hz}$ with a reduced $\chi^{2}$ of $\chi_{r}^{2}=1.57(159.0 / 101)$. The third harmonic shows a spin-up, which is not however highly significant ( $~(3 \sigma$ c.l.). The peak mass accretion rate deduced is quite low compared to the one obtained from the bolometric flux for a source distance of 6.3(3) kpc. The source distance is about 3.6(5) kpc.

\subsubsection{Fourth harmonic}

The fourth harmonic is sporadically detected in the first 15 days of the outburst, with a fractional amplitude of $\sim 1 \%$. From the fit with the constant spin frequency derivative, we obtained $|\dot{v}|<$ $2.7) \times 10^{-13} \mathrm{~Hz} \mathrm{~s}^{-1}(2 \sigma$ c.l. $)$ with a $\chi_{r}^{2}=4.03(44.28 / 11)$. The best-fit curve is reported in Fig. 7. The obtained best-fit values are $\dot{M}_{\max }<1.6 \times 10^{-9} M_{\odot}$ year $^{-1}(2 \sigma$ c.l.) for the peak mass accretion rate with a reduced $\chi^{2}$ of $\chi_{r}^{2}=4.09(45 / 11)$.

We note that, as already done in Burderi et al. (2007) and Riggio et al. (2008), we take into account the effect of the source position uncertainty on the obtained values of $v$ and $\dot{v}$ adopting the same method described in Riggio et al. (2008). In particular, in the case of constant spin frequency derivative the uncertainties are about $\Delta v=6.1 \times 10^{-8} \mathrm{~Hz}$ in the frequency and $\Delta \dot{v}=0.72 \times$ $10^{-14} \mathrm{~Hz} \mathrm{~s}^{-1}$ in the spin frequency derivative, while in the case of the physical model the uncertainties are about $\Delta v=6.1 \times$ $10^{-8} \mathrm{~Hz}$ in the frequency and $\Delta \dot{M}=4.3 \times 10^{-11} M_{\odot} \mathrm{y}^{-1}$ in the peak accretion rate.

\subsection{Spectral variability of the pulse profile}

Emulating Papitto et al. (2010) who analysed the XMM-Newton observation of the same outburst, we analysed the energy

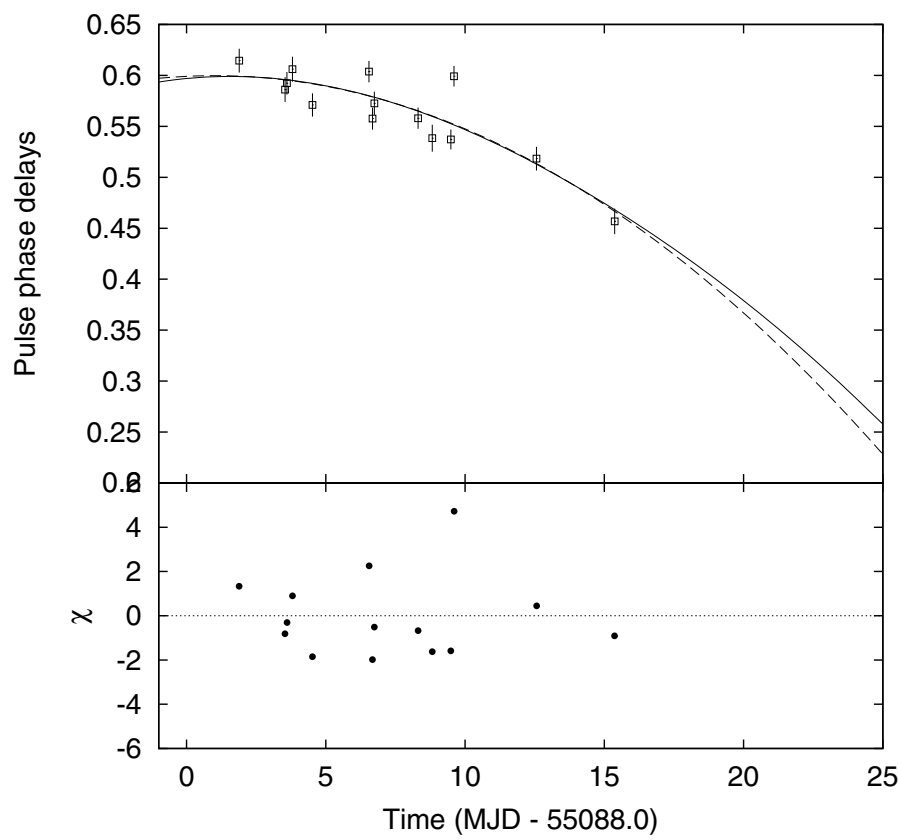

Fig. 7. In the top panel of this figure, the fourth harmonic pulse phase delays and its best-fit curves considering a constant spin frequency derivative (dashed line) and the physical model considering the material torque proportional to the flux (continuous line) are reported. In the bottom panel, we show the residuals in units of $\sigma$ with respect to the constant spin frequency derivative model.

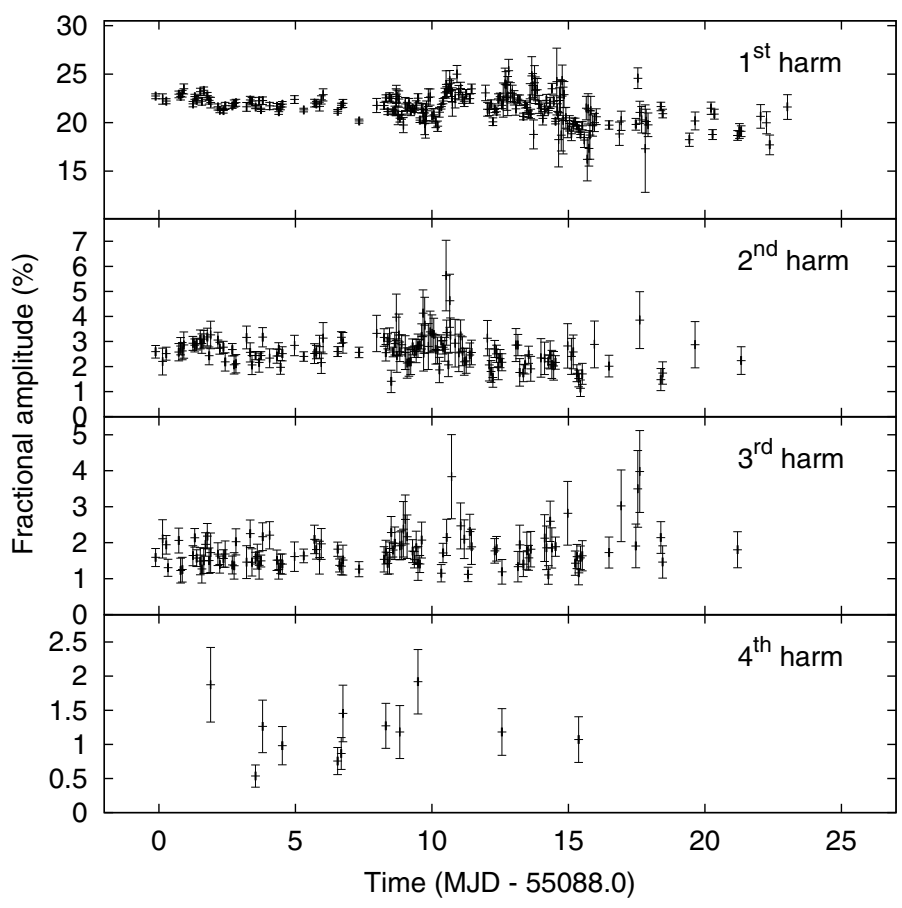

Fig. 8. The fractional amplitudes of the four harmonics are reported. Points in correspondence with the type-I bursts are not plotted due to difficulties in estimating the persistent flux.

dependence of the phase and amplitude of the three harmonic components. To achieve high quality statistics, we epoch-folded data from 55 089.233 MJD to 55 092.5379 MJD, excluding the three data files where a type-I burst was present, with an exposure time of $81 \mathrm{ks}$ and a coverage of $28 \%$. We chose this interval because the pulse phase delays have a quite stable linear trend with an average first harmonic phase scattering of 0.005 . 


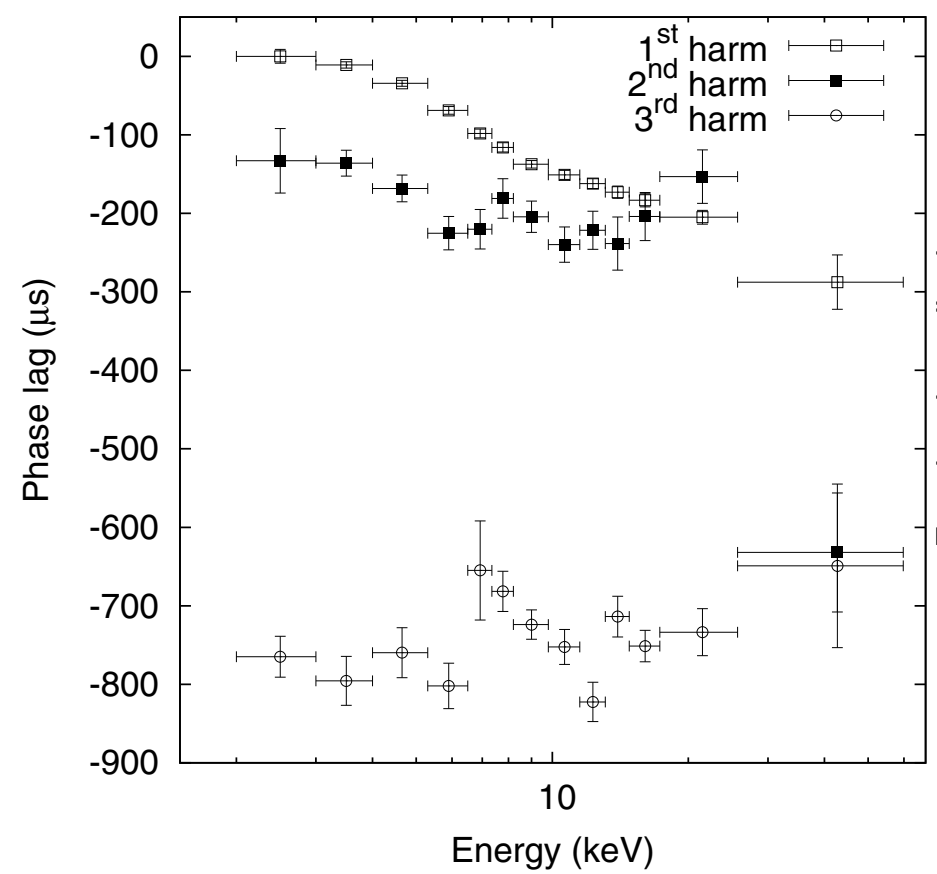

(a)

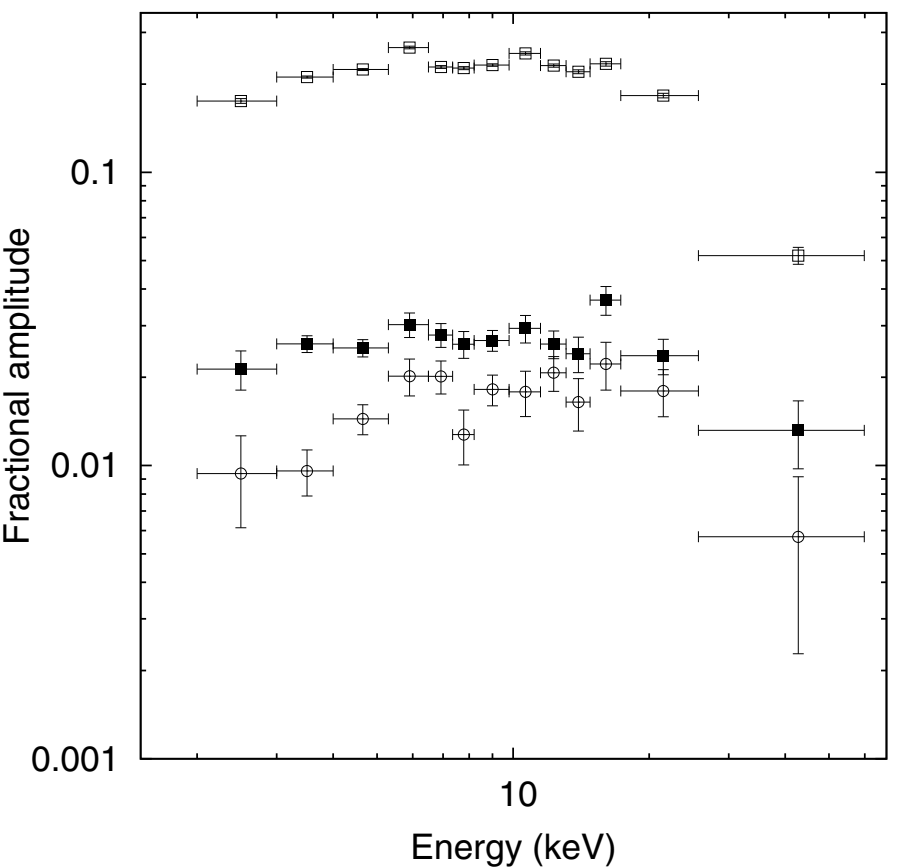

(b)

Fig. 9. In the left panel, the phase lags for the first three harmonics are reported. The phase lags are measured with respect to the maxima of each component, as done in Papitto et al. (2010). In the right panel, the fractional amplitude for the three harmonics are reported.

We consider only data from PCU2. We also evaluated the contribution to the background from the Galactic bulge, using the data described in the previous section, in the working hypothesis that the changes due to the different observational data and slightly different instrument pointing are small (see Papitto et al. 2010, for a detailed discussion). For these reasons, small systematics might be present and the confidence intervals might be underestimated. The fractional amplitude of the first harmonic (Fig. 9, right panel) increases from $17.5(3) \%$ at $2.5 \mathrm{keV}$ to $26.7(3) \%$ at $6 \mathrm{keV}$. It remains roughly constant around $23 \%$ up to $12 \mathrm{keV}$, where the fractional amplitude is $25.4(3) \%$. Above $12 \mathrm{keV}$, there is a steady decline of the fractional amplitude, although up to the energy band $25-60 \mathrm{keV}$ it is still clearly detectable with a fractional amplitude of 5.2(3)\%. The second and third harmonic fractional amplitudes show a behaviour similar to the first harmonic one that increases with energy to a maximum (around $10-20 \mathrm{keV}$ ) of $3.7(4) \%$ and $2.2(4) \%$, respectively. We also observe a decline in the fractional amplitude above $20 \mathrm{keV}$ for these harmonics. Fractional amplitudes as a function of photon energies were not clearly detected for the fourth harmonic, probably because of the long integration time and the smearing caused by the observed fourth harmonic phase fluctuations of $\sim 0.05$ (see Fig. 7), which is a considerable fraction of the fourth harmonic period. The first harmonic displays phase lags (reported in Fig. 9, left panel). As described in Papitto et al. (2010), there is a steady decrease in the phase lag up to $\sim 10 \mathrm{keV}$, where a break is clearly visible. Beyond $10 \mathrm{keV}$, the pulse phase lag still decreases, but at a lower rate.

The second harmonic shows a different trend with respect to the first harmonic. It reaches the maximum lag around $10 \mathrm{keV}$, shows no time lag at $25 \mathrm{keV}$, but then experiences a sudden jump of $\sim-450 \mu$ s between 25 and $50 \mathrm{keV}$.

The third harmonic phase lags are roughly constant throughout the energy band. We note that in the energy band
25.7-59.8 keV the phase lags for the second and third harmonic coincide.

\subsection{Type-l burst timing}

We performed a timing analysis of all the 10 type-I bursts detected in the observation, with the goal of studying the pulse profile evolution during the burst. In Fig. 10, we show the results for the second and fifth type-I bursts, the most finely sampled of the bursts in the RXTE observation. The starting date of these bursts is $55089.721 \mathrm{MJD}$ (TDB) and 55 094.619 MJD (TDB), and the decay time is 8.0(1) s and 8.5(1) s, respectively. We divided each burst in chunks holding (roughly) the same number of events so that in each folded profile a fractional amplitude of $\sim 20 \%$ can be easily detectable. We folded each chunk using 8 phase bins and performed an harmonic decomposition using only the first harmonic. The results of this analysis are reported in Fig. 10. During both bursts, the pulse phase delays remain stable and, with the exception of the very first seconds, locked to the pulse phase delays during the persistent emission (see Fig. 10, mid panel). The fractional amplitude behaviour is even more interesting because it is, within the errors, quite constant during both bursts and locked at the pre-burst value (Fig. 10, bottom panel). A detailed spectral and temporal analysis of all these type-I bursts was reported by Altamirano et al. (2010), although with different techniques.

\section{Discussion}

Among the AMPs known, IGR J17511-3057 displays several peculiarities. The highest first harmonic fractional amplitude of the source has a peak value (background corrected) at the beginning of the outburst of about $23 \%$ that linearly decrease to $17 \%$ at the end of the outburst (see Fig. 8), while the highest ever observed 


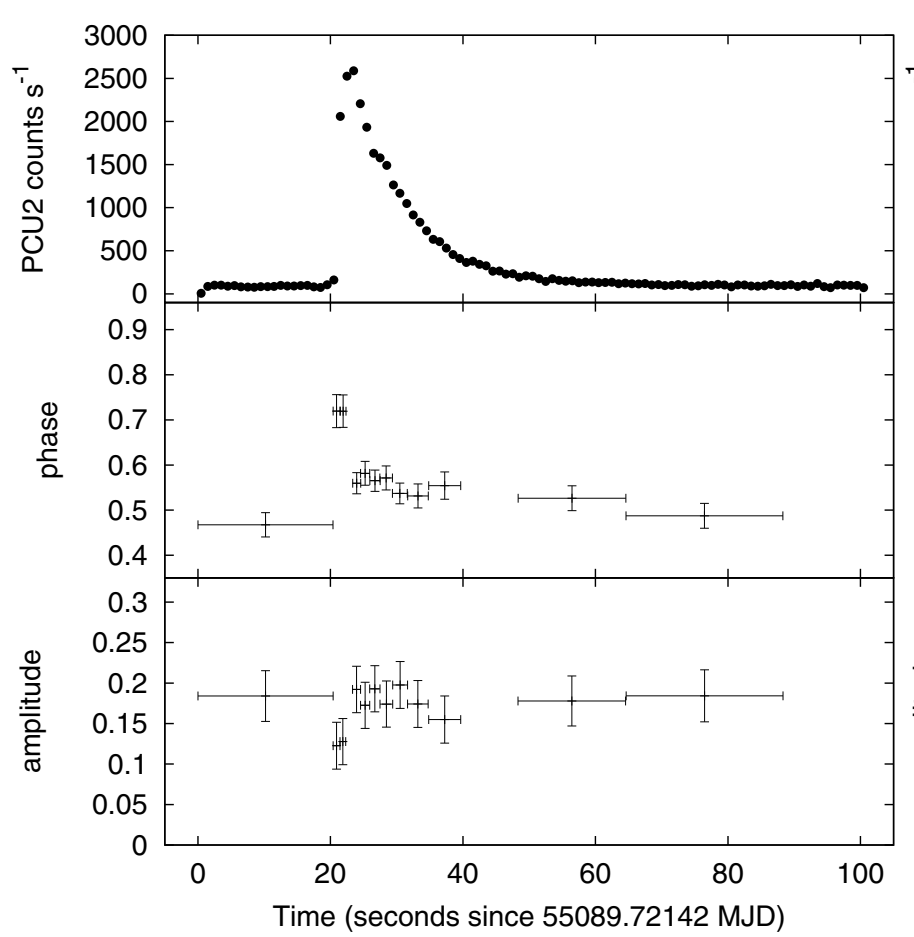

(a)

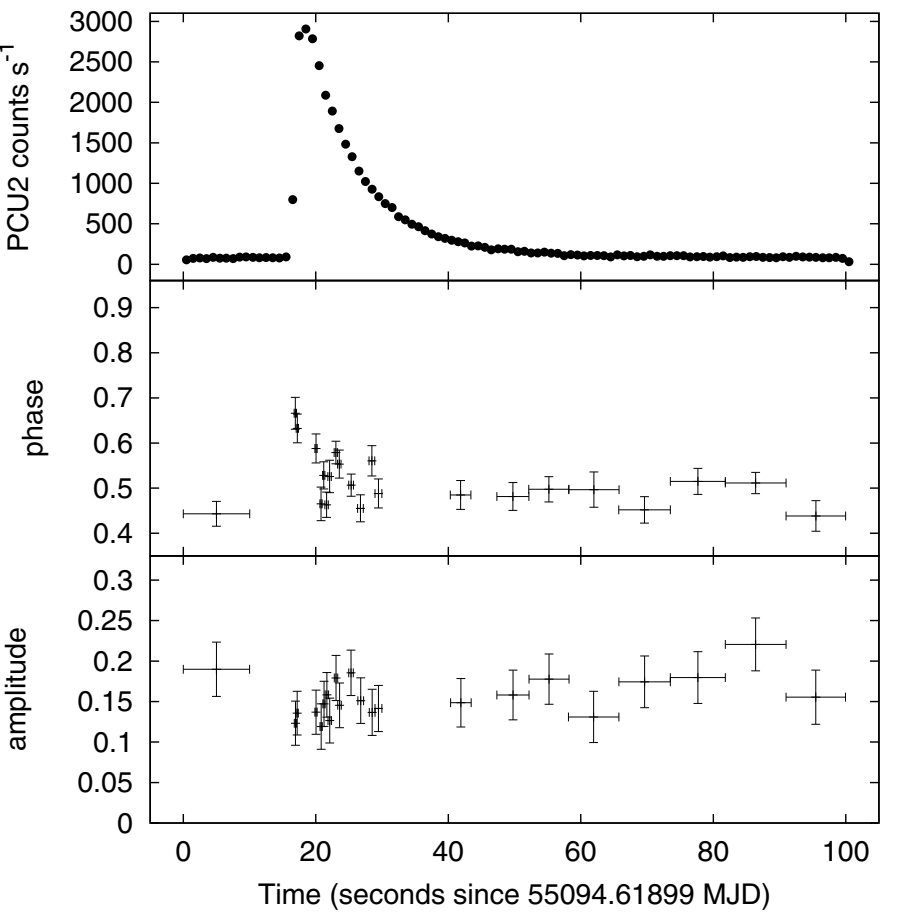

(b)

Fig. 10. Two type-I burst timing analysis is reported. For each burst in the top panel, the PCU2 count rate of the type-I burst present in this observation, in the middle panel the pulse phase delays and in the bottom panel the fractional amplitude are reported.

fractional amplitude was observed by Patruno et al. (2010) in XTE J1807-294.

The pulse shape is complex, showing, on integration times of $3 \mathrm{ks}$, a second harmonic, a third harmonic and sporadically a fourth harmonic with fractional amplitudes of $2.5 \%, 1.6 \%$, and $1 \%$, respectively (see Fig. 8). A third harmonic as strong as the second harmonic could be, following Poutanen \& Beloborodov (2006), an indication that both hot spots are visible with the secondary spot only partially visible, since for a single spot the third harmonic should be much smaller than the second harmonic $\left(a_{2} / a_{3} \gtrsim 5\right.$, Poutanen \& Beloborodov 2006), suggesting intermediate values for the inclination angle. In the AMPs, Hartman et al. (2008) reports a sporadically detectable third harmonic in SAX J1808.4-3658, while Patruno et al. (2010) detected sporadically a third and a fourth harmonic in XTE J1807-294.

That in IGR J17511-3057, the third harmonic is visible for nearly all the outburst with a total of 105 detections over 216 folded pulse profiles makes this source peculiar.

Moreover, while the fractional amplitude of the first harmonic clearly shows a steady decrease with the flux, this decrease is less evident in the second and third harmonics, for which the fractional amplitude remains more stable when the X-ray flux decreases (see Fig. 8).

However, the most interesting and puzzling result is the different behaviours of the phase delays for the four harmonic components. In particular, as for the other AMPs, such as SAX J1808.4-3658 and XTE J1807-294 (see e.g. Burderi et al. 2007; Hartman et al. 2008, 2009; Riggio et al. 2008; Patruno et al. 2010), the first harmonic has clear phase fluctuations (see Fig. 4). A discussion about the results based on the analysis of the first harmonic is strongly affected by these phase fluctuations. We can only speculate about the nature of these fluctuations. The amplitude of these fluctuations, $\sim 0.05$ in phase units, may corresponds to hot spot movements of $\sim 18$ degrees, already seen in numerical simulations (see Romanova et al. 2004; Bachetti et al. 2010), although on timescales of fractions of seconds, while the observed timescales are of a few days. We also tried to interpret these phase fluctuations with the model suggested by Patruno et al. (2010). We adopted a constant spin frequency model and a linear relation between flux and pulse phase residuals. The most closely fitting correlation parameter set gave a $\chi_{r}^{2} \simeq 23$, which is clearly unacceptable. A model to describe and correctly interpret these fluctuations needs to be developed.

However, under the working hypothesis that some exchange of angular momentum between the NS and the accreting matter has to occur during X-ray outbursts, when the accretion rate is at its maximum, we try to interpret the behaviour of the phase delays of the second and third harmonic, which appear to be less affected by phase fluctuations. That the phase delays derived from the second harmonic appear more stable than those derived from the first harmonic has been observed, for instance, during the 2002 outburst from SAX J1808.4-3658. Burderi et al. (2007) showed that, while the first harmonic phase delays clearly show a phase shift at days 14 from the beginning of the 2002 outburst, a similar phase shift was not present in the phase delays derived from the second harmonic. A similar behaviour was also observed by Riggio et al. (2008) for the AMP XTE J1807-294, which went onto outburst just once in 2003 in the RXTE era. In both these cases, the interpretation of the phase delays derived from the second harmonic in terms of accretion torques provided reasonable spin frequency derivatives (and inferred mass accretion rates onto the NS), although a different interpretation was given for both sources by Hartman et al. (2009); Patruno et al. (2009). These results have naturally to be taken with great caution, since phase fluctuations may still affect phase delays derived from the second (or higher) harmonic.

For IGR J17511-3057, again, the second harmonic shows a more regular behaviour with respect to the first harmonic and 
suggests a spin-up of the NS (see Fig. 5). From the fit with our simplified torque model, we obtain an $\dot{M}$ estimate, fixing $\mu=0$, of $\dot{M}_{\max }=0.92(10) \times 10^{-9} M_{\odot}$ year $^{-1}$, which would be compatible with the observed X-ray flux from the source if we placed the source at a distance of $6.3 \mathrm{kpc}$. This could indirectly suggest that the second harmonic component is a better tracer of the spin frequency evolution, even if a physical model that explains all the phenomenology observed in all the AMPs still needs to be developed. Results obtained on the second harmonic give, as in the case of the AMPs SAX J1808.4-3658 (Burderi et al. 2006; Hartman et al. 2009) and XTE J1807-294 (Riggio et al. 2008), reasonable values for the physical parameters of the system.

However, if we consider the third harmonic we find that it shows a spin-up that is not however highly significant $(\gtrsim 3 \sigma)$ and smaller than the value inferred for the second harmonic. The lower limit to the accretion rate $\dot{M}_{\max }=3.1(9) \times 10^{-10} M_{\odot}$ year $^{-1}$ implies a distance of 3.6(5) kpc, at $\sim 5 \sigma$ based on the value inferred from the second harmonic. This value is not compatible with the second harmonic one. The lack of literature and observations of the third harmonic behaviour in other AMPs does not allow us to compare with other cases, leaving this question open.

A fourth harmonic was detected sporadically during the outburst. We attempted to fit the pulse phase delays with the same models adopted for the other harmonics but the result was inconclusive. We note that the fourth harmonic is affected by phase fluctuations $(\sim 0.02)$ that are comparable to the first harmonic's fluctuations. It is probable that integrating on long timescales, the large fluctuations make the detection of the fourth harmonic unfeasible.

We performed a high-resolution timing analysis of all the type-I bursts present in this $R X T E$ observation. The results are very similar for all the bursts, and we show bursts 2 and 5 (see Fig. 10) since these are the most finely sampled in the RXTE observation. The first harmonic phase delays appear to rise in correspondence with the rapidly rising phase of the burst, implying that there had been a frequency drift during the first few seconds after the burst onset (Altamirano et al. 2010), and a return to the phase value during the persistent emission during the burst decay, giving some evidence that the burst probably starts not far from the hot spot in the polar cap. The fractional amplitude in each burst instead remains locked (within the errors) with the persistent emission value during all the burst, suggesting surprisingly that the temperature gradient does not vary during the burst. An interpretation of this behaviour is beyond the scope of this work.

From the orbital ephemeris reported in Table 1, the pulsar mass function is $f_{\mathrm{X}}=1.070854(21) \times 10^{-3} M_{\odot}$. From this value of mass function, we can derive a minimum mass for the companion star of $0.14 M_{\odot}$, considering an inclination angle of $90^{\circ}$ and a NS mass of $1.4 M_{\odot}$. For this minimum mass, the companion star of IGR J17511-3057 is one of the more massive companion stars among the AMPs, together with XTE J1807-294 and SAX J1748.9-2021 (Altamirano et al. 2008). Using the relation $R_{\mathrm{RL}_{2}}=1.2 \times 10^{10} m_{2,0.1}^{1 / 3} P_{2 \mathrm{~h}}^{2 / 3} \mathrm{~cm}$ (Paczyński 1971), where $m_{2,0.1}$ is the companion mass in $0.1 M_{\odot}$ units and $P_{2 \mathrm{~h}}$ is the orbital period in two-hour units, we obtain for the companion's Roche lobe radius a value of $0.248 R_{\odot}$. This value is larger than expected for a low-mass main sequence star (see Chabrier \& Baraffe 2000, 5 Gyr track), for which the corresponding radius is about $0.15 R_{\odot}$. It can be shown that the contact condition between Roche lobe and companion star pose a firm lower limit to the inclination of the system of $\sim 20$ degrees, corresponding to a companion mass of $\sim 0.45 M_{\odot}$. For smaller inclination angles, the companion star would overfill its Roche lobe. This obviously excludes the companion star being a white dwarf or an heliumcore star, while strongly suggesting that the companion star is a main sequence star, possibly bloated as a consequence of its evolutionary history (Podsiadlowski et al. 2002) or slightly evolved (Tutukov \& Iungelson 1987). The nature of the companion star is discussed thoroughly in Papitto et al. (2010).

Acknowledgements. We thank the anonymous referee for having help us to greatly improve the paper.

We also thank Sergey B. Popov for several fruitful discussions.

This work is supported by the Italian Space Agency, ASI-INAF I/088/06/0 contract for High Energy Astrophysics.

\section{References}

Altamirano, D., Casella, P., Patruno, A., Wijnands, R., \& van der Klis, M. 2008, ApJ, 674, L45

Altamirano, D., Watts, A., Linares, M., et al. 2010, MNRAS, 409, 1136

Bachetti, M., Romanova, M. M., Kulkarni, A., Burderi, L., \& di Salvo, T. 2010, MNRAS, 403, 1193

Baldovin, C. 2009, The Astronomer's Telegram, 2196, 1

Bevington, P. R., \& Robinson, D. K. 2003, Data reduction and error analysis for the physical sciences, 3rd Ed. (McGraw-Hill)

Bozzo, E., Ferrigno, C., Falanga, M., et al. 2010, A\&A, 509, L3

Burderi, L., Di Salvo, T., Menna, M. T., Riggio, A., \& Papitto, A. 2006, ApJ, 653, L133

Burderi, L., Di Salvo, T., Lavagetto, G., et al. 2007, ApJ, 657, 961

Chabrier, G., \& Baraffe, I. 2000, ARA\&A, 38, 337

Chenevez, J., Kuulkers, E., Beckmann, V., et al. 2009, The Astronomer's Telegram, 2235, 1

Deeter, J. E., Pravdo, S. H., \& Boynton, P. E. 1981, ApJ, 247, 1003

Friedman, B., \& Pandharipande, V. R. 1981, Nuclear Physics A, 361, 502

Galloway, D. K., Chakrabarty, D., Morgan, E. H., \& Remillard, R. A. 2002, ApJ, 576, L137

Ghosh, P., Pethick, C. J., \& Lamb, F. K. 1977, ApJ, 217, 578

Hartman, J. M., Patruno, A., Chakrabarty, D., et al. 2008, ApJ, 675, 1468 Hartman, J. M., Patruno, A., Chakrabarty, D., et al. 2009, ApJ, 702, 1673

Jahoda, K., Markwardt, C. B., Radeva, Y., et al. 2006, ApJS, 163, 401

Leahy, D. A., Elsner, R. F., \& Weisskopf, M. C. 1983, ApJ, 272, 256

Markwardt, C. B., Altamirano, D., Strohmayer, T. E., \& Swank, J. H. 2009a, The Astronomer's Telegram, 2237, 1

Markwardt, C. B., Altamirano, D., Swank, J. H., et al. 2009b, The Astronomer's Telegram, 2197, 1

Miller-Jones, J. C. A., Russell, D. M., \& Migliari, S. 2009, The Astronomer's Telegram, 2232, 1

Nowak, M. A., Paizis, A., Wilms, J., et al. 2009, The Astronomer's Telegram, 2215,1

Paczyński, B. 1971, ARA\&A, 9, 183

Pandharipande, V. R., \& Ravenhall, D. G. 1989, in Nuclear Matter and Heavy Ion Collisions, ed. M. Soyeur, H. Flocard, B. Tamain, \& M. Porneuf, NATO ASIB Proc, 205, 103

Papitto, A., Menna, M. T., Burderi, L., di Salvo, T., \& Riggio, A. 2008, MNRAS, 383,411

Papitto, A., Riggio, A., Burderi, L., et al. 2009, The Astronomer's Telegram, 2220, 1

Papitto, A., Riggio, A., di Salvo, T., et al. 2010, MNRAS, 407, 2575

Patruno, A., Wijnands, R., \& van der Klis, M. 2009, ApJ, 698, L60

Patruno, A., Hartman, J. M., Wijnands, R., Chakrabarty, D., \& van der Klis, M. 2010, ApJ, 717, 1253

Podsiadlowski, P., Rappaport, S., \& Pfahl, E. D. 2002, ApJ, 565, 1107

Poutanen, J., \& Beloborodov, A. M. 2006, MNRAS, 373, 836

Rappaport, S. A., Fregeau, J. M., \& Spruit, H. 2004, ApJ, 606, 436

Riggio, A., di Salvo, T., Burderi, L., et al. 2007, MNRAS, 382, 1751

Riggio, A., Di Salvo, T., Burderi, L., et al. 2008, ApJ, 678, 1273

Riggio, A., Papitto, A., Burderi, L., et al. 2009, The Astronomer's Telegram, 2221, 1

Romanova, M. M., Ustyugova, G. V., Koldoba, A. V., \& Lovelace, R. V. E. 2004, ApJ, 610, 920

Torres, M. A. P., Jonker, P. G., Steeghs, D., Simon, J. D., \& Gutowski, G. 2009, The Astronomer's Telegram, 2216, 1

Tutukov, A. V., \& Iungelson, L. R. 1987, Ap\&SS, 130, 15

van den Heuvel, E. P. J. 1984, J. Astrophys. Astron., 5, 209

Zdziarski, A. A., Johnson, W. N., \& Magdziarz, P. 1996, MNRAS, 283, 193

Życki, P. T., Done, C., \& Smith, D. A. 1999, MNRAS, 309, 561 